Pacific Journal of Mathematics

SQUARE INTEGRABLE REPRESENTATIONS AND THE
FOURIER ALGEBRA OF A UNIMODULAR GROUP 


\title{
SQUARE INTEGRABLE REPRESENTATIONS AND THE FOURIER ALGEBRA OF A UNIMODULAR GROUP
}

\section{Giancarlo MAUCERI}

\begin{abstract}
Let $G$ be a unimodular group, and let $\lambda_{d}$ be the subrepresentation of the left regular representation $\lambda$, which is the sum of the square integrable representations. The purpose of this paper is to study the representation $\lambda_{d}$ with special emphasis on the closed subspace $A_{d}(G)$ of the Fourier algebra $A(G)$ of the group which is generated by the coefficients of $\lambda_{d}$. In the last part of the paper we study in detail a particular noncompact group for which $\lambda=\lambda_{d}$.
\end{abstract}

We denote, as in [4], by $A(G)$ the algebra of the coefficients of $\lambda$, that is the algebra of continuous functions on $G$ of the type $(\lambda(x) f, g)$, with $f, g \in L^{2}(G)$. The first section contains results of a general nature: we show that $A_{d}(G)$ is the dual space of a $C^{*}$-algebra contained in $V N(G)$, the von Neumann algebra generated by the operators $\lambda(x), x \in G$, and that its unit ball is the weak closure of the extreme points of the unit ball of $A(G)$. We also show that $A_{d}(G) \subset L^{2}(G)$ if and only if the formal degrees of the square integrable representations of $G$ are bounded away from zero.

In the last section we make a closer study of an example due to J. Fell of a noncompact group $G$ for which $A_{d}(G)=A(G)$. We show that the traces of the square integrable representations of this group are bounded measures and we construct a kind of Dirichlet kernels, which also turn out to be bounded measures.

We prove that summation with respect to these kernels converges in $L^{p}(G)$ for $1<p<\infty$, but not for $L^{1}(G)$.

We conclude the paper with some remarks on the Wiener-Pitt phenomenon for bounded measures on this group.

1. We refer the reader to [4] for the definitions and the properties of the Fourier algebra $A(G)$ and the Fourier-Stieltjes algebra $B(G)$ of a locally compact group $G$, and to [3] for the basic facts about $C^{*}$-algebras, von Neumann algebras and square integrable representations of unimodular groups. Throughout the paper "group" will always mean "locally compact unimodular group" and "representation" will mean "unitary continuous representation."

Following Arsac [1], given a representation $\pi$ of $G$ on a Hilbert space $H_{\pi}$, we denote by $A_{\pi}$ the closed subspace of $B(G)$ spanned by the coefficients of $\pi$, i.e., the functions $(\pi(x) \mu \mid \nu), x \in G, \mu, \nu \in H_{\pi}$. Let $\lambda_{d}$ be the subrepresentation of the left regular representation of $G$ 
which is the sum of all the irreducible square integrable representations of $G$. Then $A_{d}(G)=A_{\lambda_{d}}$ is a closed subspace of $A(G)$. From the results of [1] it follows easily that there exists a closed subspace $A_{c}(G) \subset A(G)$ such that $A(G)=A_{d}(G) \oplus A_{c}(G)$. Moreover $A_{d}(G)$ itself is the direct sum $\bigoplus_{\pi \in \hat{G}_{d}} A_{\pi}$, where $\widehat{G}_{d}$ denotes the family of all equivalence classes of irreducible square integrable representations of $G$.

Now, given a representation $\pi$ of $G$ on the Hilbert $H_{\pi}$, we denote by $\bar{\pi}$ its conjugate representation on $\bar{H}_{\pi}$ the Hilbert space conjugate to $H_{\pi}$. We remember that $A(G)$ is endowed with a structure of left $V N(G)$-module [3, Prop. 3.17]. For $\pi \in \widehat{G}_{d}$ let $P_{\pi}$ and $K_{\pi}$ denote respectively the minimal central projection and the minimal biinvariant subspace corresponding to $\pi$. Then the following facts are a more or less immediate consequence of [3, Ch. 14]. For every $\pi \in \widehat{G}_{d} A_{\pi}=$ $P_{\bar{\pi}} A(G)$ is contained in $K_{\pi}$. Moreover the mapping $u \rightarrow d_{\pi} \pi(u)$, where $d_{\pi}$ is the formal degree of $\pi$ and $\pi(u)=\int_{G} \pi(x) u(x) d x$, is an isometric isomorphism of $A_{\bar{\pi}}$ onto the Banach space $T C\left(H_{\pi}\right)$ of all trace class operators on $H_{\pi}$. Any function $u \in A_{d}(G)$ is the sum of its Fourier series:

$$
u(x)=\sum_{\pi \in \hat{\epsilon}_{d}} d_{\pi} \operatorname{tr}\left(\pi\left(x^{-1}\right) \pi(u)\right)
$$

where the series converges absolutely as well as in $A(G)$.

If $G$ is a compact abelian group it is well known that its dual group $\hat{G}$ is a discrete measure space. Therefore $A(G)$, being isometric to $l^{1}(\hat{G})$, can be identified with the dual of the Banach space $c_{0}(\hat{G})$ of all bounded complex functions on $\hat{G}$, vanishing at infinity. The following lemma shows that for $G$ nonabelian a similar result holds for $A_{d}(G)$.

Lemma 1.1. Let $c_{0}\left(\widehat{G}_{d}\right)$, be the direct sum, in the $C^{*}$-algebra theoretical sense, of the algebras $C_{\pi}^{*}=\pi\left(C^{*}(G)\right)$ for all $\pi \in \hat{G}_{d}$. Then $A_{d}(G)$ can be isometrically identified with the dual space of $c_{0}\left(\widehat{G}_{d}\right)$ via the following pairing:

$$
\langle T, u\rangle=\sum_{\pi \in \hat{G}_{d}} d_{\pi} \operatorname{tr}(\pi(T) \pi(u))
$$

for $T \in c_{0}\left(\hat{G}_{d}\right), u \in A_{d}(G)$.

Proof. For every $\pi \in \widehat{G}_{d}, C_{\pi}^{*}$ is isometric to the $C^{*}$-algebra $L C\left(H_{\pi}\right)$ of all compact operators on $H_{\pi}\left[3,4.1 .11\right.$, and 18.4.1]. Since $A_{\bar{\pi}}$ is isometric to $T C\left(H_{\pi}\right)$, which is the dual of $L C\left(H_{\pi}\right)$, the lemma easily follows.

Now, to find the extreme points of the unit ball of $A(G)$, we need the following lemma. 
Lemma 1.2. Let $T$ be an operator in the unit ball of the space $T C(H)$ of trace class operators on a Hilbert space $H$. Then these are equivalent:

(i) $T$ is an extreme point.

(ii) $|T|$ is a projection of rank one.

(iii) There exist $\mu, \nu \in H,|\mu|=|\nu|=1$ such that $T \phi=(\dot{\phi} \mid \nu) \mu$ for every $\dot{\phi} \in H$.

Proof. Let us denote by $T C_{1}(H)$ the unit ball of the space $T C(H)$. It is obvious that (ii) and (iii) are equivalent. We prove only the equivalence between (i) and (ii). Let $T$ be an extreme point in $T C_{1}(H)$ and suppose that there exist $R, S$ in $T C_{1}(H)$ such that $|T|=1 / 2(R+S)$; then $1=1 / 2(\operatorname{tr}(R)+\operatorname{tr}(S))$.

It follows that $\operatorname{tr}(R)=\operatorname{tr}(S)=1$, so $R$ and $S$ are positive operators.

Since $|T|=1 / 2(R+S)$ and since $T$ is an extreme point we get: $T=U R=U S$. Then $|T|=U^{*} U R=R$ and $T=U^{*} U S=S$ because $U^{*} U$ is greater than or equal to the supports of $R$ and $S$. So $|T|$ is an extreme point of $T C_{1}(H)$. Since $T C(H)$ is the dual space of the $C^{*}$-algebra $L C(H)[3,4.1 .2]$, the extreme points in its positive unit ball are just zero and the pure states, i.e., the positive operators $P$ in $T C_{1}(H)$ such that $0 \leqq P^{\prime} \leqq P$ implies $P^{\prime}=\lambda P, 0 \leqq \lambda \leqq 1$ $[3,2.5 .5]$. This proves that $|T|$ must be a projection of rank one. So (i) implies (ii).

To show that (ii) implies (i) we shall prove that if $P$ is a projection of rank one and $U$ is a partial isometry such that $U^{*} U=P$, then $U P$ is an extreme point in $T C_{1}(H)$. Since the final projection of $U$ is one-dimensional there is an isometry $W$ which coinc:des with $U$ on its support. Therefore $U P=W P$. Now let $R, S$ be in $C T_{1}(H)$ such that $W P=1 / 2(R+S)$; then $P=1 / 2\left(W^{*} R+W^{*} S\right)$ and $W^{*} R, W^{*} S \in$ $T C_{1}(H)$. Snce $P$ is one-dimensional, $P$ defines a pure state on $T C(H)$. Therefore $P$ is an extreme point in $T C_{1}(H)$ and $P=$ $W^{*} R=W^{*} S$.

So $U P=W P=R=S$ and $U P$ is extreme in $T C_{1}(H)$.

THeOREM 1.1. Let $u$ be in the unit ball of $A(G)$. Then $u$ is an extreme point if and only if there exist $\pi \in \widehat{G}_{d}$ and vectors $\mu, \nu \in$ $H_{\pi},|\boldsymbol{\mu}|=|\boldsymbol{\nu}|=1$, such that $u(x)=(\pi(x) \mu \mid \nu)$. Moreover $A_{d}(G)$ is the closed subspace of $A(G)$ spanned by the extreme points of the unit ball of $A(G)$.

Proof. Let $u$ be an extreme po nt in the unit ball of $A(G)$. Let $P \in V N(G)$ be the central support of $u$, considered as an ultraweakly continuous form on $V N(G)$. We claim that $P$ is a minimal central 
projection in $V N(G)$. Suppose to the contrary that there exists a central projection $Q$ in $V N(G)$ such that $0<Q<P$. Let $\alpha=\|Q u\|_{A}$ and $\beta=\|(P-Q) u\|_{A}$. Then $\alpha+\beta=1$ and $\alpha>0, \beta>0$ because $P$ is the minimal central projection such that $P u=u$. Hence $u=\alpha u_{1}+$ $\beta u_{2}$, where $u_{1}=Q u /\|Q u\|_{A}$ and $u_{2}=(P-Q) u /\|(P-Q) u\|_{A}$ are in the unit ball of $A(G)$. But this contradicts the extremality of $u$. Hence there exists $\pi \in \widehat{G}_{d}$ such that $u$ is an extreme point in the unit ball of $A_{\pi}$. Therefore $d_{\pi} \bar{\pi}(u)$ is an extreme point in the unit ball of $T C\left(H_{\pi}\right)$. Then, by Lemma 1.2, there exist $\mu, \nu \in \bar{H}_{\pi},|\mu|=|\nu|=1$, such that $d_{\pi} \bar{\pi}(u) \phi=(\phi \mid \mu) \nu$ for every $\phi \in \bar{H}_{\pi}$. We may assume that $\bar{\pi}$ is a subrepresentation of the left regular representation $\lambda$ of $G$. Then, denoting by $[\cdot \mid \cdot]$ the inner product in $H_{\pi}$ and by $(\cdot \mid \cdot)$ the inner product in $\bar{H}_{\pi}$, and remembering that $(\phi \mid \psi)=[\psi \mid \phi]$, we have by $[3,14.3 .3]$ :

$$
\begin{aligned}
\int_{G} u(x)(\bar{\pi}(x) \phi \mid \psi) d x=(\bar{\pi}(u) \phi \mid \psi) & =d_{\pi}^{-1}(\phi \mid \mu) \overline{(\psi \mid \nu)} \\
\times \int_{G}(\pi(x) \phi \mid \psi) \overline{(\bar{\pi}(x) \mu \mid \nu)} d x & =\int_{G}(\bar{\pi}(x) \phi \mid \psi) \overline{[\pi(x) \mu \mid \nu]} d x
\end{aligned}
$$

for every $\phi, \psi \in \bar{H}_{\pi}$. Since, by $[3,14.3 .1]$, the functions $(\pi(x) \dot{\phi} \mid \psi)$, $\phi, \psi \in \bar{H}_{\pi}$ are dense in $K_{\bar{\pi}}$, the identity $u(x)=[\pi(x) \mu \mid \nu]$ follows at once. The last assertion of the theorem follows by Lemma 1.1 and the Krein Milman theorem.

As we have seen in the introductory remarks, $A_{\pi} \subset L^{2}(G)$ for every $\pi \in \widehat{G}_{d}$. It is natural to ask whether $A_{d}(G)$ is contained in $L^{2}(G)$ or not. Since we have that $\|u\|_{A}=\sum_{\pi} d_{\pi} \operatorname{tr}(|\pi(u)|)$ for every $u \in A_{d}(G)$ and $|f|_{2}^{2}=\sum_{\pi} d_{\pi} \operatorname{tr}\left(\pi(f)^{*} \pi(f)\right)$ for every $f \in \oplus K_{\pi}, \pi \in \widehat{G}_{d}$, a comparison of the two formulas, together with the closed graph theorem, yields at once that $A_{d}(G)$ is contained in $L^{2}(G)$ if and only if the formal degrees of the square integrable representations of $G$ are bounded away from zero.

It is well known that if $G$ is a locally compact abelian group then $A(G)=A_{d}(G)$ if and only if $G$ is compact. If $G$ is not abelian the situation is more complicated, because there exist noncompact groups such that $A(G)=A_{d}(G)$. In the next section we shall study in detail an example of such groups. A more detailed discussion of the structure and properties of unimodular groups, whose regular representation is the direct sum of irreducible subrepresentations, will appear in a forthcoming paper of M. Picardello and the author [7]. Here we bound ourselves to the following few remarks.

REMARK 1. Let $G$ be a noncompact unimodular group such that 
$A(G)=A_{d}(G)$. Then $\inf \left\{d_{\pi}: \pi \in \widehat{G}_{d}\right\}=0$. This is an easy consequence of the remark following Theorem 1.1 and the fact $A(G)$ cannot be contained in $L^{2}(G)$, because $G$ is noncompact [9].

REMARK 2. Let $G$ be as before and let $K$ be a compact normal subgroup of $G$. Then $G / K$ is again a noncompact unimodular group whose regular representation is the direct sum of its irreducible components. Indeed $G / K$ is clearly unimodular and noncompact and $A(G / K)$ is isometric to the biinvariant closed selfadjoint subalgebra of $A(G)$ of the functions which are constant on $K$-cosets [4]. It follows easily that $A(G / K)=A_{d}(G / K)$.

We conclude this section with a result which is related to the contents of the last remark but not to the main theme of the paper. If $G$ is any locally compact group and $K$ is a compact normal subgroup, the functions of $A(G)$ which are constant on the cosets of $K$ form a closed biinvariant selfadjoint subalgebra of $A(G)$. The fact that viceversa every closed biinvariant selfadjoint subalgebra of $A(G)$ is of this type is a special case of a result of M. Takesaki and N. Tatsuuma [Duality and subgroups, Annals of Math., v. 93 (1971) 344-364, Theorem 9]. It can also be deduced from the following theorem which is a slight improvement of a result of [1]. We believe that our proof, shorter than that of [1] can also shed light on the result of Takesaki and Tatsuuma.

Let $\mathfrak{A}$ be a nonzero right invariant closed selfadjoint subalgebra of $A(G)$. Then by [11] there exists a projection $P \in V N(G)$ such that $\mathfrak{A}=P A(G)$. Let $H_{P}=P\left(L^{2}(G)\right)$ be the corresponding subspace of $L^{2}(G)$.

THEOREM 1.2. The space $H_{P}$ is closed under multiplication by functions of $\mathfrak{X}$. If $\mathfrak{A}$ separates the points of $G$ then $\mathfrak{A}=A(G)$.

Proof. First we claim that $\mathfrak{A} \cap H_{P}$ is dense in $H_{P}$. Indeed let $f$ be any function in $H_{P}$ and let $\phi_{\alpha}$ be an approximated identity for the convolution of continuous functions with compact support in $G$. Then $\phi_{\alpha} * f$ is in $A(G) \cap L^{2}(G)$ and $\lim \phi_{\alpha} * f=f$ in $L^{2}(G)$. Since $P\left(\phi_{\alpha} * f\right) \in \mathfrak{A} \cap$ $H_{P}$, the claim is proved. Now let $u \in \mathfrak{A}, f \in H_{P}$ and let $\left\{f_{\alpha}\right\}$ be a net in $\mathfrak{A} \cap H_{P}$ converging to $f$ in $L^{2}(G)$. Then $u f_{\alpha} \in \mathfrak{A} \cap H_{P}$, because $u$ is a bounded function and $\mathfrak{A}$ is an algebra. Since $\lim u f_{\alpha}=u f$, we have $u f \in H_{P}$.

To prove the last assertion of the theorem, observe that if $\mathscr{A}$ separates the points of $G$, by the Stone-Weierstrass theorem, $\mathfrak{A}$ is uniformly dense in the space $C_{0}(G)$ of continuous functions on $G$ vanishing at infinity. Therefore $H_{P}$ is also closed under the multiplication by 
functions in $C_{0}(G)$. Let $g$ be any nonzero continuous function in $H_{P}$. Such function actually exists, because $\mathfrak{A} \cap H_{P}$ is dense in $H_{P}$. Let $\mathscr{C}$ be any open set on which $g$ is bounded away from zero. Now let $f$ be any continuous function with compact support in $\mathscr{C}$ and denote by $h$ the function so defined: $h(x)=f(x) / g(x)$ for $x \in \mathscr{U}, h(x)=0$ for $x \notin \mathscr{Q}$. Then $h \in C_{0}(G)$ and $f=h g$ is in $H_{P}$. Hence $H_{P}$ contains the space $C_{c}(\mathscr{C})$ of the continuous functions with compact support in $\mathscr{U}$. Applying the translation invariance of $H_{P}$ and a simple partition of unity argument, it is easy to see that $C_{c}(G) \subset H_{P}$. Therefore $H_{P}=L^{2}(G)$. Hence $P=I$ and $\mathfrak{A}=A(G)$.

2. Fell's example. In [2] L. Baggett describes the following example, due to Fell of group $G$ such that $A(G)=A_{d}(G)$.

Let $p$ be a prime number, $N$ the $p$-adic numbers field, $K$ the subset of $p$-adic numbers $k$ whose valuation $|k|_{p}$ is one. $K$ is a compact abelian group w.r.t. multiplication. For $n \in N, k \in K$ set $k(n)=k n$. Then $K$ acts as group of automorphisms of the additive group $N$. The orbits of $N$ under the action of $K$ are $\{0\}$ and $N_{j}=\{n: n \in N$, $\left.|n|_{p}=p^{-j}\right\}, j \in Z$. Let $G=K \circ N$ be the semidirect product of $K$ and $N$. Then $G$ is a regular semidirect product because $\hat{N}=N=$ $\left(\cup_{j \in Z} N_{j}\right) \cup\{0\}$. Therefore using the representation theory of group extensions [6], we can describe the irreducible representations of $G$.

One verifies that $\hat{G}$ is the union of two sets $\hat{G}_{1}=\left\{\pi_{j}: j \in Z\right\}$, $\widehat{G}_{2}=\left\{\pi_{\theta}: \theta \in \hat{K}\right\}$. The representations in $\hat{G}_{1}$ can be realized on the Hilbert space $L^{2}(K)$, while the representations in $\widehat{G}_{2}$ are one-dimensional.

If $\pi_{j} \in \widehat{G}_{1}$ and $f \in L^{2}(K)$, then:

$$
\left[\pi_{j}(l, m) f\right](k)=\exp \left(2 \pi i p^{j} k m\right) f(k l)
$$

for $(l, m) \in G, k \in K$. Here the exponential of a $p$-adic number

$$
n=p^{j} \sum_{i \geqq 0} n_{i} p^{i}, 0 \leqq n_{\imath}<p,
$$

is defined as follows:

$$
\exp (2 \pi i n)= \begin{cases}\exp \left(2 \pi i p^{j} \sum_{i+j<0} n_{i} p^{i}\right) & \text { for } j<0 \\ 1 & \text { for } j \geqq 0 .\end{cases}
$$

If $\pi_{\theta} \in \widehat{G}_{2},(l, m) \in G$, then $\pi_{\theta}(l, m)=\theta(l)$.

Figà-Talamanca in [5] proved that when $A(G) \neq A_{d}(G)$ there exist positive definite continuous functions which vanish at infinity but are not in $A(G)$. He also asked whether or not unimodularity alone is sufficient to prove the existence of such functions for $G$ noncompact.

The following corollary answers in the negative to this question. Recall first that the Fourier-Stieltjes algebra $B(G)$ is the algebra, 
under pointwise operations of all linear combinations of continuous positive definite functions on $G[4] . B(G)$ is also the Banach involution algebra of the coefficients $u(x)=(\pi(x) \xi \mid \eta), \xi, \eta \in H_{\pi}$ of all unitary continuous representations of $G$, normed thus:

$$
\|u\|_{B}=\min \{|\xi||\eta|: u(x)=(\pi(x) \xi \mid \eta)\} .
$$

Corollary 2.1. $B(G)$ is the direct sum $A(G) \oplus A P(G)$, where $A P(G)$ is the Banach involution algebra of the almost periodic functions on $G$. In particular a function $u \in B(G)$ vanishes at infinity if and only if $u \in A(G)$.

Proof. Since $\hat{G}$ is countable an arbitrary unitary representation $\pi$ of $G$ is the direct sum (rather than the direct integral) $\pi=\left(\bigoplus_{j \in Z} n_{j} \pi_{j}\right) \oplus\left(\bigoplus_{\theta \in \hat{K}} n_{\theta} \pi_{\theta}\right)$. Therefore if $u$ is any coefficient of $\pi, u$ decomposes into the sum $u=u_{1}+u_{2}, u_{1} \in A(G)$ and $u_{2}=\sum_{\theta \in \hat{K}} n_{\theta} \theta$, where the series converges in $B(G)$, and hence uniformly. Thus $u_{2}$ is an almost periodic function [3, 16.2.1. (v)]. If $u$ vanishes at infinity $u_{2}, \bar{u}_{2}$ and hence $\left|u_{2}\right|^{2}$ vanish at infinity. Since $A P(G)$ is a Banach involution algebra with respect to pointwise operations and complex conjugation, $\left|u_{2}\right|^{2}$ is an almost periodic function whose mean is zero. Hence $u_{2}=0[3,16.3]$.

We shall now evaluate the "diagonal" coefficients of the representation $\pi_{j} \in \widehat{G}_{d}$, with respect to the orthonormal basis $\hat{K}$ in $L^{2}(K)$. This will enable us to compute the formal degrees of the square integrable representations of $G$ and to study the convergence of the Fourier series for functions in $L^{p}(G), 1 \leqq p<+\infty$.

Lemma 2.2. For $\pi_{j} \in \widehat{G}_{d}$ denote by $\phi_{\theta \theta}^{(j)}(l, m)=\left(\pi_{j}(l, m) \theta \mid \theta\right)$ the coefficient of $\pi_{j}$ corresponding to $\theta \in \hat{K}$. Then $\phi_{\theta \theta}^{(j)}(l, m)=\phi^{(j)}(m) \theta(l)$, where:

$$
\phi^{(j)}(m)=\left\{\begin{array}{ccl}
1 & \text { for } & |m|_{p} \leqq p^{j} \\
\frac{1}{1-p} & \text { for } & |m|_{p}=p^{j+1} \\
0 & \text { for } & |m|_{p}>p^{j+1}
\end{array}\right.
$$

The formal degrëe of $\pi_{j}$ is $d_{j}=p^{-j}[(p-1) / p]^{2}$.

Proof. We have for $(l, m) \in G$ :

$$
\dot{\phi}_{\theta, \theta}^{(j)}(l, m)=\left(\pi_{j}(l, m) \theta \mid \theta\right)=\int_{K} \exp \left(2 \pi i p^{j} k m\right) \theta(k l) \theta(k) d_{k}(k)
$$

where $d_{k}(k)$ denotes the Haar measure on $K$ which coincides with the Haar measure $d_{N}(k)$ on $N$, since $d_{N}(m, k)=|m|_{p} d_{N}(k)$ for every $m \in N$. 
Then:

$$
\phi_{\theta, \theta}^{(j)}(l, m)=\theta(l) \int_{K} \exp \left(2 \pi i p^{j} k m\right) d_{N}(k) \quad(l, m) \in G .
$$

By a change of variable, setting $p^{j} k m=t$ and $|m|_{p}=p^{\mu}$, the integral

$$
\begin{aligned}
& I=\int_{K} \exp \left(2 i \pi p^{j} k m\right) d_{N}(k) \text { becomes: } \\
& I=p^{j-\mu} \int_{N_{j-\mu}} \exp (2 \pi i t) d_{N}(t) .
\end{aligned}
$$

So we need to compute, for every relative integer $s$, the integral:

$$
I_{s}=\int_{N_{s}} \exp (2 \pi i t) d_{N}(t) \text {. }
$$

Let $t$ be in $N_{s}$; then $t=p^{s} \sum_{n=0}^{+\infty} t_{n} p^{n}$ where $0 \leqq t_{n}<p$ for $n \in N$, and $t_{0} \neq 0$. Then:

$$
\exp (2 \pi i t)=\left\{\begin{array}{lll}
\exp \left(2 \pi i p^{s} \sum_{0 \leqq i<-s} t_{n} p^{n}\right) & \text { for } & s<0 \\
1 & \text { for } & s \geqq 0 .
\end{array}\right.
$$

Therefore for $s \geqq 0, I_{s}$ is just the measure of $N_{s}=p^{s} K$, i.e., $I_{s}=p^{-s}$. For $s=-1$ we have:

$$
I_{-1}=\sum_{j=1}^{p-1} \int_{N_{-1, j}} \exp \left(2 \pi i j p^{-1}\right) d_{N}(t)=\sum_{j=1}^{p-1} \exp \left(2 \pi i j p^{-1}\right) \int_{N_{-1, j}} d_{N}(t)
$$

where $N_{-1, j}=\left\{t: t \in N_{-1}, t_{0}=j\right\}$. Since $N_{-1}$ is the disjoint union of the $N_{-1, j}$ for $j=1, \cdots, p-1$ and $N_{-1, j}=N_{-1, i}+j-i$ for $i, j=1, \cdots$, $p-1$ :

$$
\int_{N_{-1}, j} d_{N}(t)=\frac{1}{p-1} \int_{N_{-1}} d_{N}(t)=\frac{p}{-p-1}
$$

for every $j=1, \cdots, p-1$.

So

$$
I_{-1}=\frac{p}{p-1} \sum_{j=1}^{p-1} \exp \left(2 \pi i j p^{-1}\right)=\frac{-p}{p-1} .
$$

Now for $s \leqq-2$, let $J$ be the set of multiindices $j=\left(j_{0}, j_{1}, \cdots, j_{1-s}\right)$ s.t. $0 \leqq j_{l}<p$ for $1=0, \cdots, 1-s$ and $j_{0} \neq 0$. Setting $\langle j, p\rangle=$ $\sum_{1=0}^{1-s} j_{l} p^{l}$ we have:

$$
I_{s}=\sum_{j \in J} \exp \left(2 \pi i p^{s}\langle j, p\rangle\right) \int_{N_{s}, j} d_{N}(t)
$$

where $N_{s, j}=\left\{t: t \in N_{s}, t_{0}=j_{0}, t_{1}=j_{1}, \cdots, t_{1-s}=j_{1-s}\right\}$. Since $N_{s}$ is the 
disjoint union of the $N_{s, j}, j \in J$ and $N_{s, j}=N_{s, j^{\prime}}+\langle j, p\rangle-\left\langle j^{\prime}, p\right\rangle, j$, $j^{\prime} \in J$, then

$$
\int_{N_{s, j}} d_{N}(t)=\frac{p^{-s}}{p^{1-s}(p-1)}
$$

for every $j \in J$. Therefore:

$$
I_{s}=\frac{1}{p(p-1)} \sum_{j \in . J} \exp \left(2 \pi i p^{s}\langle j, p\rangle\right)=0
$$

because

$$
\sum_{j \in J} \exp \left(2 \pi i p^{s}\langle j, p\rangle\right)=\sum_{k=0}^{p^{-s}-1} \exp \left(2 \pi i p^{s} k\right)-\sum_{k=0}^{p^{1-s}-1} \exp \left(2 \pi i p^{8-1} k\right)
$$

and

$$
\sum_{k=0}^{\alpha-1} \exp \left(2 \pi i \alpha^{-1} k\right)=0
$$

for every positive integer $\alpha$.

We have thus that:

$$
\phi_{\theta \theta}^{(j)}(l, m)=\theta(1) p^{j-\mu} I_{j-\mu}=\left\{\begin{array}{cll}
\theta(l) & \text { for }|m|_{p} \leqq p^{j} \\
\frac{1}{1-p} \theta(l) & \text { for }|m|_{p}=p^{j+1} \\
0 & \text { for }|m|_{p}>p^{j+1}
\end{array}\right.
$$

To compute the formal degree $d_{j}$ of $\pi_{j}$ it is sufficient to observe that, since $\phi^{(j)}$ is a positive definition function, whose value in the identity is one $d_{j}^{-1}=\left|\phi^{(j)}\right|^{2}=p^{j}[p / p-1]^{2}[3,14.4 .3]$.

Let $\gamma_{j}$ be the positive definite central measure on $G$, defined by $\gamma_{j}\left(f * g^{*}\right)=\operatorname{tr}\left(\pi_{j}(f) \pi_{j}(g)^{*}\right)$, for $f, g \in C_{c}(G)$ and $\pi_{j} \in \hat{G}_{1}$. If $\delta_{1}$ denotes the Dirac measure at 1 on $K$ then $\gamma_{j}=\phi^{(j)} \otimes \delta_{1}$ (here we have identified $\phi^{(j)}$ with the measure $\left.\phi^{(j)}(x) d_{N}(x)\right)$. Indeed it is easy to verify by means of Lemma 2.2 that for every $\psi \in C_{c}(N)$ and $\theta \in \hat{K}$

$$
\left(\phi^{(j)} \otimes \delta_{1}\right)(\psi \otimes \theta)=\gamma_{j}(\psi \otimes \theta) \text {. }
$$

The measure $\gamma_{j}$ is called the "character measure" of the representation $\pi_{j}[3,17.2 .4]$.

Definition 1. We define the Dirichlet Kernel $\left\{D_{n}\right\}$ by:

$$
D_{n}=\sum_{j=-n}^{n} d_{j} \gamma_{j}
$$

for every positive integer $n$.

An easy but lengthy computation shows that, for every $n, D_{n}$ 
is a measure whose total variation $\left\|D_{n}\right\|_{M}$ is bounded by the constant 2. Therefore the convolution operator $\lambda\left(D_{n}\right) f=D_{n} * f$ is a bounded operator on $L^{p}(G)$ for $1 \leqq p \leqq+\infty$. Since $\gamma_{i} * \gamma_{j}=d_{j}^{-1} \delta_{i j} \gamma_{j}$, for $i, j \in$ $Z, \lambda\left(D_{n}\right)$ is a projection, which for $p=2$ coincides with the sum $\sum_{j=-n}^{n} P_{\pi_{j}}$ of the minimal central projections associated with the square integrable representations $\pi_{j}, j=-n, \cdots, n$.

Therefore for $f \in L^{2}(G)$ :

$$
f=\sum_{\jmath \in \boldsymbol{Z}} d_{j}\left(\gamma_{j} * f\right)
$$

where the series converges in $L^{2}(G)$.

Definition 2. For every function $f \in L^{p}(G), 1 \leqq p \leqq \infty$ we call $\sum_{j \in Z} d_{j}\left(\gamma_{j} * f\right)$ the formal Fourier series of $f$. We say that a function $f \in L^{p}(G)$ is a trigonometric polynomial in $L^{p}(G)$ if

$$
D_{n} * f=\sum_{j=-n}^{n} d_{j}\left(\chi_{j} * f\right)=f
$$

for some $n$. The following theorem shows that the Dirichlet kernel is a summability kernel for $L^{p}(G) 1<p<\infty$.

THEOREM 2.3. If $f \in L^{p}(G), 1<p<\infty$, then:

$$
f=\lim _{n \rightarrow+\infty} D_{n} * f=\lim _{n \rightarrow+\infty} \sum_{j=-n}^{n} d_{j}\left(\gamma_{j} * f\right)
$$

in the $L^{p}(G)$ norm.

Proof. Assume that trigonometric polynomials are dense in $L^{p}(G)$ for $1<p<\infty$. Let $f \in L^{p}(G) \varepsilon>0$ and let $P$ be a trigonometric polynomial in $L^{p}(G)$, satisfying $|f-P|_{p}<\varepsilon / 4$. For $n$ large enough we have $D_{m} * P=P$ and hence:

$$
\left|D_{n} f-f\right|_{p} \leqq\left|D_{n} *(f-P)\right|_{p}+|P-f|_{p}<\varepsilon .
$$

Therefore it remains only to show that trigonometric polynomials are actually dense in $L^{p}(G), 1<p<\infty$. Let $g$ be a continuous function with compact support in $G$. Then $g \in L^{2}(G)$ and $\lim _{n \rightarrow+\infty} D_{n} * g=g$ in the $L^{2}(G)$ norm. On the other hand, since $\left|D_{n} * g\right|_{p} \leqq 2|g|_{p}$ and $1<p<\infty$, there exists a subsequence $\left\{D_{n_{k}} * g\right\}$ which converges in the weak topology of $L^{p}(G)$ to some limit $h$. By the Banach-Saks theorem, there is a sequence of convex combinations of the $D_{n_{k}} * g$, which converges to $h$ in the norm of $L^{p}(G)$. Taking a subsequence which converges almost everwhere, we have $g=h$ a.e. Thus trigonometric polynomials are dense in $L^{p}(G), 1<p<\infty$. 
REMARK. The Dirichlet kernel $\left\{D_{n}\right\}$ is not a summability kernel for $L^{1}(G)$. In fact let $f \in L^{1}(G)$ be a function such that

$$
\pi_{\theta_{0}}(f)=\int_{N} \int_{K} f(1, m) \theta_{0}(1) d_{N}(1) d_{N}(m) \neq 0
$$

for some $\theta_{0} \in \hat{K}$. For every function $h \in L^{1}(G)$ and for every $\theta \in \hat{K}$, we have $\lim _{j \rightarrow-\infty}\left(\pi_{j}(h) \theta \mid \theta\right)=\pi_{\theta}(h)$, by Lemma 2.2 and the dominated converge theorem. Since $\pi_{j}\left(D_{n} * h\right)=0$ for $|j|>n$, then $\pi_{\theta}\left(D_{n} * h\right)=0$ for every positive integer $n$.

Now, for every $n \in N$ :

$$
\left|D_{n} * f-f\right|_{1} \geqq\left\|\lambda\left(D_{n} * f-f\right)\right\|=\sup _{j \in Z}\left\|\pi_{j}\left(D_{n} * f-f\right)\right\| \geqq\left|\pi_{\theta_{0}}(f)\right|
$$

since $\| \pi_{j}\left(D_{n} * f-f\right)|| \geqq\left|\left(\pi_{j}\left(D_{n} * f-f\right) \theta_{0} \mid \theta_{0}\right)\right|, j \in Z$ and

$$
\lim _{j \rightarrow-\infty}\left|\left(\pi_{j}\left(D_{n} * f-f\right) \theta_{0} \mid \theta_{0}\right)\right|=\mid \pi_{\theta_{0}}(f) \text {. }
$$

This proves that $D_{n} * f$ cannot converge to $f$ in $L^{1}(G)$, if $\pi_{\hat{\theta}_{0}}(f) \neq 0$.

We conclude now our study of the group $G$ with some final remarks on the algebra $M(G)$ of all complex measures of bounded variation on $G$.

REMARK. There exists a measure $\mu \in M(G)$ such that the operator $\lambda(\mu) f=\mu * f$ for $f \in L^{2}(G)$ has inverse and yet $\mu^{-1}$ does not exist, as an element of the algebra $M(G)$. This phenomenon was first discovered in $M(\boldsymbol{R})$ by Wiener and Pitt [8], who showed that there exists a measure $\mu \in M(R)$ such that 0 is in the spectrum of $\mu$ but the Fourier-Stieltjes transform $\hat{\mu}$ of $\mu$ is bounded away from zero. Since $K$ is a compact abalian group by [10, Th. 6.4.1], there exists a measure $\nu \in M(K)$ such that $0 \in \operatorname{sp}(\nu)$ and $\hat{\nu}(\theta) \geqq 1$ for every $\theta \in \hat{K}$.

Then it is straightforward to check that if $\mu=\delta_{e}-d_{c} \phi^{(0)} \otimes\left(\delta_{1}-\nu\right)$, where $\delta_{e}$ is the Dirac measure at the identity $e=(1,0)$ of $G, 0 \in \mathrm{sp}(\mu)$. Moreover, for $j \neq 0, \pi_{j}(\mu)$ is the identity on $L^{2}(K)$ and $\pi_{0}(\mu)$ is the operator whose matrix representation with respect to the basis $\hat{K}$ in $L^{2}(K)$ is given by the diagonal matrix whose eigenvalues are the $\hat{\nu}(\theta), \theta \in \hat{K}$. Therefore $\lambda(\mu)^{-1}$ exists.

REMARK. The same construction as above, together with [10, Th. 6.4.1] can be used to show that the spectral radius of a measure $\mu$ in $M(G)$ is much larger than the spectral radius of the operator $\lambda(\mu)$. Actually, given any complex number $z_{0}$ there is a measure $\mu \in M(G)$ such that $z_{0} \in \operatorname{sp}(\mu)$ and $\|\lambda(\mu)\| \leqq 1$. (Take $\nu \in M(K)$ such that $z \in \operatorname{sp}(\nu)$ and $|\hat{\nu}(\theta)| \leqq 1$ for every $\theta \in \hat{K}$, and set $\mu=d_{0} \phi^{(0)} \otimes \nu_{\text {.) }}$

After this paper was completed we learned that Corollary 2.1 was also proved independently by M. E. Walter [12]. 


\section{REFERENCES}

1. G. Arsac, Sur l' espace de Banach engendré par les coefficientes d'une representation unitaire, Thèse presenté a l'Université Claude-Bernard, Lions-1.

2. L. Baggett, $A$ separable group having a discrete dual space is compact, J. Functional Analysis, 10 (1972), 149-158.

3. J. Dixmier, Les $C^{*}$-algebres et leurs representations, Cahiers scientifiques fasc. 29, Gauthier-Villars, Paris, (1964).

4. P. Eymard, L algebre de Fourier $d$ un groupe localment compact, Bull. Soc. Math. France, 92 (1964), 181-123.

5. A. Figà-Talamanca, Positive definite functions which vanish at infinity Pacific, J. Math., (to appear).

6. G. Mackey, Unitary representations of group extensions, I, Acta Math., 99 (1958), 265-311.

7. G. Mauceri and M. Picardello, Unimodular groups with purely atomic Plancherel measure, (to appear).

8. H. R. Pitt and N. Wiener, On absolutely convergent Fourier-Stieltjes transforms, Duke Math. J., 4 (1938), 420-436.

9. N. W. Rickert, Convolution of $L^{2}$ functions, Colloq. Math., 19 (1968), 301-303.

10. W. Rudin, Fourier Analysis on Groups, Interscience Tracts in Pure and Applied Math. 12, Interscience, New York, (1962).

11. M. Takesaki, On the conjugate space of an operator algebra, Tôhoku Math. J., 10 (1958), 194-203.

12. M. E. Walter, On a theorem of Figà-Talamanca, Proc. Amer. Math. Soc., (to appear).

Received April 13, 1977 and in revised form June 30, 1977.

Istituto Di Matematica

UNIVERSTTA DI GENOVA

ITALY 


\section{PACIFIC JOURNAL OF MATHEMATICS}

EDITORS

RICHARD ARENS (Managing Editor)

University of California

Los Angeles, California 90024

C. W. Curtis

University of Oregon

Eugene, OR 97403

C. C. MOORE

J. DUGUNDJI

Department of Mathematics University of Southern Californa Los Angeles, California 90007

R. Finn AND J. Milgram Stanford University Stanford, California 94305

University of California

Berkeley, CA 94720

\section{ASSOCIATE EDITORS}

E. F. BeCKENBACH

B. H. NeUMANN

F. WOLF

K. YosHida

\section{SUPPORTING INSTITUTIONS}

UNIVERSITY OF BRITISH COLUMBIA UNIVERSITY OF SOUTHERN CALIFORNIA CALIFORNIA INSTITUTE OF TECHNOLOGY UNIVERSITY OF CALIFORNIA MONTANA STATE UNIVERSITY UNIVERSITY OF NEVADA, RENO STANFORD UNIVERSITY UNIVERSITY OF TOKYO UNIVERSITY OF UTAH NEW MEXICO STATE UNIVERSITY WASHINGTON STATE UNIVERSITY OREGON STATE UNIVERSITY UNIVERSITY OF WASHINGTON UNIVERSITY OF OREGON OSAKA UNIVERSITY 


\section{Pacific Journal of Mathematics \\ Vol. 73, No. 1 \\ March, 1977}

Thomas Robert Berger, Hall-Higman type theorems. $V \ldots \ldots \ldots \ldots \ldots \ldots \ldots$

Frank Peter Anthony Cass and Billy E. Rhoades, Mercerian theorems via

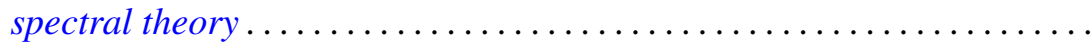

Morris Leroy Eaton and Michael David Perlman, Generating $\mathrm{O}(n)$ with

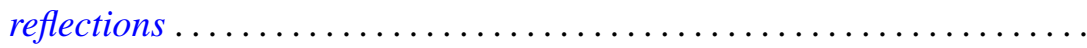

Frank John Forelli, Jr., A necessary condition on the extreme points of a

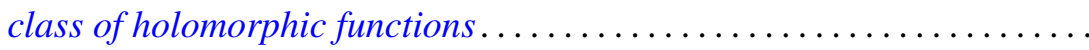

Melvin F. Janowitz, Complemented congruences on complemented

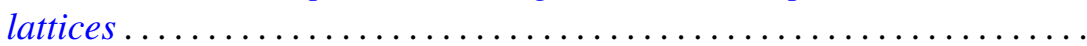

Maria M. Klawe, Semidirect product of semigroups in relation to amenability, cancellation properties, and strong $F \phi$ lner conditions....

Theodore Willis Laetsch, Normal cones, barrier cones, and the "spherical image" of convex surfaces in locally convex spaces ................

Chao-Chu Liang, Involutions fixing codimension two knots.............

Joyce Longman, On generalizations of alternative algebras .............

Giancarlo Mauceri, Square integrable representations and the Fourier

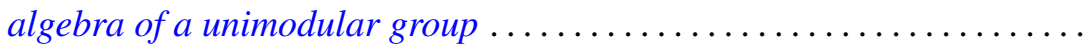

J. Marshall Osborn, Lie algebras with descending chain condition...

John Robert Quine, Jr., Tangent winding numbers and branched

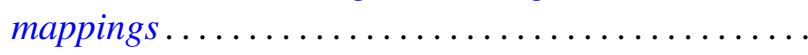

Louis Jackson Ratliff, Jr. and David Eugene Rush, Notes on ideal covers and associated primes .

H. B. Reiter and N. Stavrakas, On the compactness of the hyperspace of faces.

Walter Roth, A general Rudin-Carlson theorem in Banach-spaces ..

Mark Andrew Smith, Products of Banach spaces that are uniformly rotund in every direction.

Roger R. Smith, The R-Borel structure on a Choquet simplex ...

Gerald Stoller, The convergence-preserving rearrangements of real infinite series. ...

Graham H. Toomer, Generalized homotopy excision theorems modulo a Serre class of nilpotent groups...

Norris Freeman Weaver, Dehn's construction and the Poincaré conjecture....

Steven Howard Weintraub, Topological realization of equivariant intersection forms... 